\title{
Im Fokus: Demografie, Digitalisierung und Datensicherheit
}

\author{
Am 24. September ist Bundestagswahl. Deshalb \\ haben diverse Institutionen und Verbände das erste \\ Halbjahr dazu genutzt, ihre Forderungen an die Poli- \\ tik heranzutragen. Nachdem sich für die Zahnärzte- \\ schaft der Freie Verband Deutscher Zahnärzte (FVDZ) \\ und die Bundeszahnärztekammer (BZÄK) positioniert \\ hatten, verabschiedete im Juni die Vertreterver- \\ sammlung (VV) der Kassenzahnärztlichen Bundes- \\ vereinigung (KZBV) ihre "Agenda Mundgesundheit \\ 2017-2021". Die Standespolitik spricht damit mit \\ einer starken gemeinsamen Stimme.
}

Der KZBV-Chef Dr. Wolfgang Eßer hat die Agenda den VV-Delegierten in Köln vorgestellt. Sie umfasst die gesundheitspolitischen Positionen der Vertragszahnärzteschaft zur Sicherstellung und Weiterentwicklung der zahnmedizinischen Versorgung in Deutschland. „Ganz oben stehen dabei die drei großen D's", sagte Eßer. Die Buchstaben stehen für: Demografischer Wandel, Digitalisierung und Datensicherheit. Oberste Ziele seien die Verbesserung der Mundgesundheit und die wohnortnahe, flächendeckende und qualitativ hochwertige Versorgung, betonte Eßer. Hinsichtlich der drei D's nannte er die Herausforderungen für die Zahnärzteschaft: „Wir müssen den demografischen Wandel bewältigen, die Chancen der Digitalisierung nutzen und zugleich die Datensicherheit gewährleisten." Die Digitalisierung müsse dringend genutzt werden, „um den Bürokratiewahnsinn in den Praxen zu minimieren, damit die Kollegen endlich wieder mehr Zeit für die Patienten bekommen.“

Grundsätzlich zielt die Agenda auf ein pluralistisches Gesundheitswesen mit freiberuflichen Strukturen, freier Arztwahl der Patienten, einem selbstverwalteten System sowie einer Honorierung ab, mit der Zahnärzte in ihren Praxen betriebswirtschaftlich arbeiten können. Mit diesen Forderungen weiß sich die KZBV in guter Gesellschaft, denn auch die BZÄK und der FVDZ verfolgen diese Ziele mit Nachdruck und haben entsprechende Positionspapiere erarbeitet.

Die Positionen der „Agenda Mundgesundheit 2017-2021“ sind in einem Zwölf-Punkte-Plan zusammengefasst und auf der Website der KZBV zu lesen. Die Vertreterversammlung als oberstes Entscheidungsgremium der Vertragszahnärzteschaft in Deutschland, in dem die Vorstände der einzelnen KZVen und ihre Stellvertreter sitzen, verabschiedete die Agenda einhellig.

\section{Telematik: „Fristen sind nicht einzuhalten“}

Im Gegensatz zu Eßer, der schon vor seiner Wiederwahl im März Vorsitzender des KZBV-Vorstandes war, berichteten seine neu gewählten Stellvertreter Dr. Karl-Georg Pochhammer und Martin Hendges zum ersten Mal, womit sie sich in ihren neuen Ämtern in den vergangenen Monaten beschäftigt haben. Pochhammer, der unter anderem für das Thema Telematik zustän-

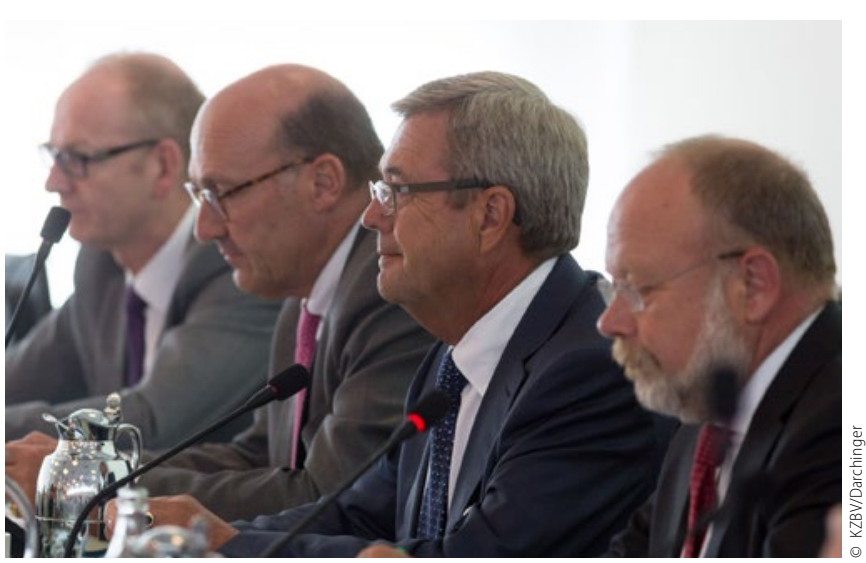

Vorstand und Vorsitzender der VV

dig ist, versuchte, die Aufregung rund um den Aufbau einer Telematikinfrastruktur in deutschen Arzt- und Zahnarztpraxen etwas zu relativieren. „Die vorgegebenen Fristen sind nicht einzuhalten“, sagte Pochhammer. „Durch die Verschiebung des bundesweiten Online-Rollouts müssen auch alle anderen Termine verschoben werden." Laut E-Health-Gesetz sind niedergelassene Ärzte, Zahnärzte und Psychotherapeuten dazu verpflichtet, ab 1. Juli 2018 flächendeckend die Daten der Versicherten auf der elektronischen Gesundheitskarte über mobile Kartenterminals online zu prüfen und zu aktualisieren. Wenn die Spitzenorganisationen der Leistungserbringer und Kostenträger die gesetzlich festgeschriebene Frist nicht einhalten, drohen Kürzungen der Haushaltsmittel und Vergütungsabschläge.

Pochhammer zeigte sich jedoch davon überzeugt, dass es so schlimm nicht kommt: „Es kann sicher keine Sanktionen mehr geben“, sagte er und verwies auf einen Beschluss der Gesellschafterversammlung. Auch auf die Frage eines Delegierten, wie sich Zahnärzte momentan verhalten sollten, antwortete Pochhammer unaufgeregt und rief zu Geduld statt Aktionismus auf. Derzeit könne abgewartet werden, es liefen weiterhin Gespräche mit dem Bundesgesundheitsministerium, berichtete der KZBVVize. „Wir sollten die Kollegen beruhigen, dass sie nicht überstürzt irgendwelche Geräte kaufen."

\section{MVZ: KZBV fordert fairen Wettbewerb}

Der zweite stellvertretende KZBV-Chef Martin Hendges berichtete unter anderem über Pläne im Bereich der Medizinischen Versorgungszentren (MVZ). Die KZBV wolle unter Einbeziehung eines Schiedsamtes gegen die Ungleichbehandlung von niedergelassenen Zahnärzten und MVZ vorgehen, erklärte er. Um einen fairen Wettbewerb zu ermöglichen, müssten die einseitigen Beschränkungen für Niedergelassene aufgehoben werden. Derzeit dürfen MVZ unbegrenzt Zahnärzte anstellen. Im Gegensatz dazu ist es einem Vertragsarzt- oder -zahnarzt in klassischen Kooperationsformen oder der Einzelpraxis lediglich erlaubt, zwei Zahnärzte zu beschäftigen. Hendges kündigte an, dass die KZBV eine Änderung des Manteltarifvertrages verhandeln möchte. 